\title{
BDNF Exerts Contrasting Effects on Peripheral Myelination of NGF-Dependent and BDNF-Dependent DRG Neurons
}

\author{
Junhua Xiao, ${ }^{1}$ Agnes W. Wong, ${ }^{1}$ Melanie M. Willingham, ${ }^{1}$ Selma K. Kaasinen, ${ }^{5}$ Ian A. Hendry, ${ }^{5}$ Jason Howitt, ${ }^{2}$ \\ Ulrich Putz, ${ }^{2}$ Graham L. Barrett, ${ }^{4}$ Trevor J. Kilpatrick, ${ }^{1,3}$ and Simon S. Murray ${ }^{1,3}$ \\ ${ }^{1}$ Neurotrophin Signaling Laboratory, Centre for Neuroscience, ${ }^{2}$ Brain Development Laboratory and ${ }^{3}$ Multiple Sclerosis Research Group, Florey \\ Neuroscience Institutes, and ${ }^{4}$ Molecular Neurobiology Laboratory, Department of Physiology, The University of Melbourne, Melbourne, Victoria 3010, \\ Australia, and ${ }^{5}$ Developmental Neurobiology Laboratory, John Curtin School of Medical Research, Australian National University, Canberra, Australian \\ Capital Territory 0200, Australia
}

\begin{abstract}
Although brain-derived neurotrophic factor (BDNF) has been shown to promote peripheral myelination during development and remyelination after injury, the precise mechanisms mediating this effect remain unknown. Here, we determine that BDNF promotes myelination of nerve growth factor-dependent neurons, an effect dependent on neuronal expression of the p75 neurotrophin receptor, whereas BDNF inhibits myelination of BDNF-dependent neurons via the full-length TrkB receptor. Thus, BDNF exerts contrasting effects on Schwann cell myelination, depending on the complement of BDNF receptors that are expressed by different subpopulations of dorsal root ganglion neurons. These results demonstrate that BDNF exerts contrasting modulatory roles in peripheral nervous system myelination, and that its mechanism of action is acutely regulated and specifically targeted to neurons.
\end{abstract}

\section{Introduction}

During development of the peripheral nervous system (PNS), reciprocal interactions between axons and Schwann cells (SCs) are crucial for the coordinated generation of myelin, an essential component of a functional PNS. Several neuronally derived molecules influence myelination, including neuregulins (Taveggia et al., 2005), glial cell line-derived neurotrophic factor (Höke et al., 2003), and neurotrophins (Cosgaya et al., 2002; Chan et al., 2004).

The neurotrophin family compromises nerve growth factor (NGF), brain-derived neurotrophic factor (BDNF), neurotrophin-3 (NT-3), and NT-4/5. Two classes of receptors mediate neurotrophin signaling: the tropomyosin-related kinase (Trk) receptors and the p75 neurotrophin receptor (p75NTR) (Huang and Reichardt, 2001). Analysis of neurotrophin and neurotrophin receptor knock-out mice have established that neurotrophins are essential for PNS development. Dorsal root ganglion neurons (DRG) are particularly sensitive to the neurotrophins, with $\sim 80 \%$ dependent on NGF for survival, 30\% dependent on BDNF and $\sim 20 \%$ on NT-3 (Wright and Snider, 1995; Conover and Yancopoulos, 1997). The neurotrophins also regulate peripheral myelination (Chan et al., 2001, 2004; Cosgaya et al., 2002). However, the cellular and molecular basis of how neuro-

Received Aug. 11, 2008; revised Feb. 18, 2009; accepted Feb. 21, 2009.

This work was supported by the Australian National Health and Medical Research Council (NH\&MRC), Multiple Sclerosis Research Australia (MSRA), and the University of Melbourne (UoM). J.X. is the recipient of an NH\&MRC/ MSRA Betty Cuthbert Fellowship (454330), a UoM Early Career Researcher grant, and an MSRA project grant. S.S.M. is the recipient of NH\&MRC Project Grant 400341 and an MSRA project grant.

Correspondence should be addressed to Dr. Simon S. Murray, Centre for Neuroscience, The University of Melbourne, Melbourne, Victoria 3010, Australia. E-mail: ssmurray@unimelb.edu.au.

DOI:10.1523/JNEUROSCI.3811-08.2009

Copyright $\odot 2009$ Society for Neuroscience $\quad$ 0270-6474/09/294016-07\$15.00/0 trophins regulate myelination of the various DRG subpopulations remains poorly understood. NGF enhances Schwann cell myelination of NGF-dependent neurons via activation of neuronal TrkA receptors but has no effect on BDNF-dependent neurons which do not express TrkA (Chan et al., 2004). NT-3 inhibits peripheral myelination in vitro (Chan et al., 2001; Cosgaya et al., 2002), whereas analysis of NT3-/- mice suggests it exerts a promyelinating influence (Woolley et al., 2008). BDNF enhances peripheral myelination during development and remyelination after injury (Zhang et al., 2000; Chan et al., 2001; Cosgaya et al., 2002; Song et al., 2006). BDNF can be transported along and secreted by DRG axons to enhance Schwann cell myelination and induce Schwann cell polarity, a necessary step in the induction of myelination (Chan et al., 2006; Ng et al., 2007). However, it remains unclear whether BDNF acts directly on Schwann cells or indirectly via neurons to promote myelination and whether BDNF similarly influences myelination of different DRG neuron subpopulations.

Here, we demonstrate that BDNF exerts contrasting influences on peripheral myelination: enhancing myelination of NGF-dependent DRG neurons but inhibiting myelination of BDNF-dependent neurons. These influences are exerted via neuronally expressed receptors: activation of p75NTR has a promyelinating influence, whereas activation of TrkB is inhibitory.

\section{Materials and Methods}

Postnatal day 2 (P2) Sprague Dawley rats, NGFR/SVJ-129 (p75NTR-/-) and SVJ-129 wild-type mice were used for DRG and SC culture. p75NTR $-/-$ mice were obtained from a colony previously shown to be $95 \%$ congenic to the inbred SVJ-129 strain (Greferath et al., 2000) and subsequently back-crossed a further 12 generations.

DRG-SC cocultures were established as described previously (Chan et al., 2004). Briefly, NGF-dependent DRGs were purified in the presence of 


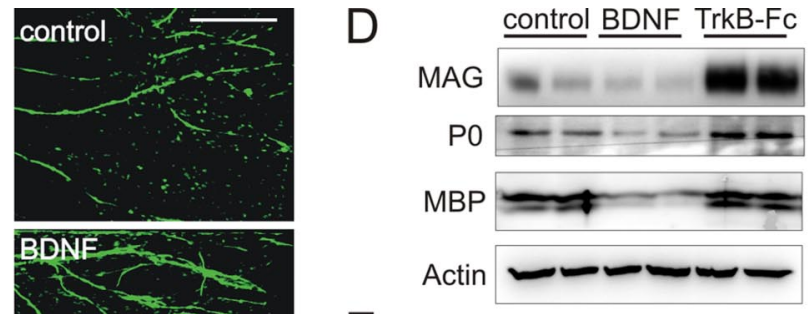

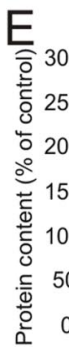

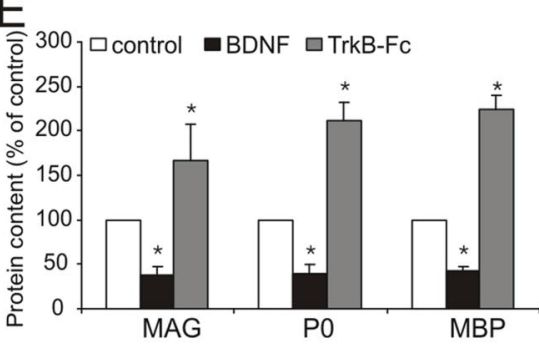

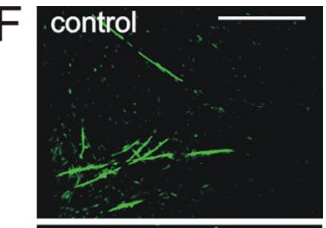


Figure 1. BDNF exerts contrasting influences on myelination of NGF-dependent and BDNF-dependent DRG neurons. $\boldsymbol{A}-\boldsymbol{C}, \mathrm{BDNF}$ enhances myelination of NGF-dependent neurons. $\boldsymbol{A}, \boldsymbol{B}$, Western blot $(\boldsymbol{A})$ including densitometric analysis ( $\boldsymbol{B}$; mean $\pm \mathrm{SEM}, n=3$ ) of myelinating cocultures containing NGF-dependent neurons cultured with either exogenous BDNF (100 ng/ml) or TrkB-Fc (1 $\mu \mathrm{g} / \mathrm{ml}$ ) and probed for MAG, P0, and MBP with actin as a control. Exogenous BDNF increased myelin expression, whereas TrkB-Fc inhibited their expression ( ${ }^{*} p<0.05$ vs control). $C$, MBP immunostaining of parallel cultures. Compared with controls, a qualitative increase in MBP + myelin segments occurred with BDNF and a decrease with TrkB-Fc. Scale bar, $100 \mu m$. $D-F$, BDNF inhibits myelination of BDNF-dependent neurons. $\boldsymbol{D}$, Western blot including densitometric analysis ( $\boldsymbol{E}$; mean $\pm S E M, n=3$ ) of cocultures containing BDNF-dependent neurons. Exogenous BDNF reduced, whereas TrkB-Fc enhanced the expression of MAG, P0, and MBP $\left({ }^{*} p<0.01\right.$ vs control). $F, M B P$ immunostaining of parallel cultures. Compared with controls, a qualitative decrease in $\mathrm{MBP}+$ myelin segments occurred with BDNF, whereas an increase occurred with TrkB-Fc. Scale bar, $100 \mu \mathrm{m}$.

NGF (100 ng/ml) and BDNF-dependent DRGs with BDNF (100 ng/ml) for 2-3 weeks. Myelination of DRG-SC cocultures was initiated with ascorbic acid $(50 \mu \mathrm{g} / \mathrm{ml})$ and treated as indicated.

Campenot chambers (CAMP\#3; Tyler Research) were used as described previously (Campenot, 1992; Chan et al., 2004; Ishibashi et al., 2006; Ng et al., 2007). DRGs were plated into the central compartment and axons allowed to extend into the outer compartments. SCs were seeded into the outer compartment, myelination induced, and chambers treated as indicated. Immunocytochemistry was used as a direct quantitative readout for myelination.

Lentiviral particles were prepared from p75NTR-shRNA and controlscrambled shRNA plasmids (Open Biosystems) by transfection into HEK293 cells. SCs were infected with concentrated lentiviral particles for $48 \mathrm{~h}$, then seeded onto DRGs. p75NTR knockdown efficacy was determined by Western blot.

Western blot analysis of DRG-SC cocultures for myelin-associated glycoprotein (MAG; Millipore Bioscience Research Reagents), myelin basic protein (MBP; Millipore Bioscience Research Reagents), myelin protein zero (P0; Abnova), and p75NTR (Promega) was performed in duplicate for each coculture; blots shown are representative of three independent experiments. The optical density value for each band was determined using National Institutes of Health ImageJ, corrected to a loading control, then normalized against the control condition. Alternatively, cocultures were fixed with $4 \%$ paraformaldehyde and myelin segments visualized with anti-MBP (Millipore Bioscience Research Reagents) on a Zeiss confocal microscope. For analysis of Campenot chambers, $\mathrm{MBP}+$ myelin segments were counted in four random images.

\section{Results}

\section{BDNF enhances myelination of NGF-dependent neurons}

We first examined the role BDNF plays in modulating myelination of NGF-dependent DRG neurons by either providing exogenous BDNF or by removing endogenous BDNF using the BDNF "scavenger" TrkB-Fc. BDNF enhanced myelin formation by Western blot analysis of coculture lysates for MAG, MBP, and P0 (Fig. 1A). Densitometric analyses indicate BDNF increased P0 and MBP expression, whereas TrkB-Fc decreased it (Fig. 1B). MBP immunostaining was used to assess myelin segment formation and was potentiated by BDNF (Fig. $1 C$ ). The promyelinating effect of BDNF did not increase either Schwann cell proliferation or differentiation in vitro (supplemental Fig. 1, available at www.jneurosci.org as supplemental material).

\section{BDNF inhibits myelination of BDNF-dependent neurons}

DRG neurons are heterogeneous with respect to their neurotrophin dependence. Because full-length TrkB receptors (TrkB-FL) are absent from NGF-dependent neurons (supplemental Fig. 2, available at www.jneurosci.org as supplemental material) (Wright and Snider, 1995; Chan et al., 2004), we more comprehensively investigated the influence of BDNF on myelination and established cocultures using BDNF-dependent DRG neurons. In contrast to the promyelinating effect observed with NGFdependent neuron cocultures, BDNF reduced myelination of BDNF-dependent neurons, whereas TrkB-Fc increased myelin formation. Both qualitative (Fig. $1 D$ ) and quantitative (Fig. $1 E$ ) Western blot analysis demonstrate a reduction in MAG, P0, and MBP expression by BDNF, whereas an increase with TrkB-Fc. These changes were reflected by qualitative assessment of MBP immunostained cocultures (Fig. $1 F$ ).

These data demonstrate that BDNF exerts contrasting influences on Schwann cell myelination, promoting myelination of NGF-dependent DRG neurons, but inhibiting myelination of BDNF-dependent DRG neurons.

BDNF enhances myelination of NGF-dependent neurons via a neuronal mechanism

Given that NGF-dependent neurons, BDNF-dependent neurons, and Schwann cells all express receptors for BDNF (supplemental Fig. 2, available at www.jneurosci.org as supplemental material), it remained uncertain how BDNF influenced myelination. To address this issue, we investigated myelination using compartmentalized Campenot chambers (Campeonet, 1992). This involves establishing DRG-SC cocultures in isolated compartments, permitting addition of factors to chambers containing solely the DRG soma and proximal axons ("DRG soma"), or distal axon plus Schwann cells (Axon +SC), then using immuno- 
cytochemistry as a quantitative readout for myelination (Fig. 2A) (Chan et al., 2004; $\mathrm{Ng}$ et al., 2007). A liquid impermeable but axonal permissive barrier separates chambers, the integrity of which was tested quantitatively by monitoring phenol red diffusion between compartments. Medium collected was measured for phenol red absorption at $560 \mathrm{~nm}$ (supplemental Fig. 3, available at www.jneurosci.org as supplemental material). Only chambers that displayed both significant axon growth and no sign of leakage were analyzed.

We first investigated the influence of BDNF on NGF-dependent neurons (Fig. $2 B, C)$. Compared with control cocultures (condition 1), BDNF applied to the Axon + SC compartment increased the number of myelinated segments (condition 2), whereas TrkB-Fc inhibited myelination (condition 3). Importantly, BDNF added to the DRG soma compartment produced a robust increase in myelin formation (condition 4), indicating it is sufficient for BDNF to act solely on neurons to promote myelination. Interestingly, adding TrkB-Fc to the neuronal compartment (condition 5) had no effect on myelination, suggesting that either Schwann cells are the source of endogenous BDNF, or that BDNF is anterogradely transported and secreted from axons.

\section{BDNF activates neuronal p75NTR receptors to promote myelination}

Thus, both NGF and BDNF promote myelination of NGFdependent neurons via activation of neuronally expressed receptors. In the case of NGF, activation of TrkA is responsible (Chan et al., 2004). As BDNF does not activate TrkA and these neurons do not express TrkB-FL (supplemental Fig. 2, available at www. jneurosci.org as supplemental material), this indicates that NGF and BDNF influence myelination through fundamentally different mechanisms. NGF-dependent neurons express two receptors for BDNF: p75NTR and truncated TrkB receptor 1 (TrkB-T1) (supplemental Fig. 2, available at www.jneurosci.org as supplemental material). Interestingly, analysis of the p75NTR -/ - mice has identified that p75NTR acts as a positive modulator of peripheral myelination; however, the cell type responsible is unknown (Bentley and Lee, 2000; Cosgaya et al., 2002; Song et al., 2006). To investigate whether neuronal p75NTR receptors mediate the promyelinating influence of BDNF, we used a mixed species coculture, using NGF-dependent neurons isolated from p75NTR -/- mice (Lee et al., 1992) (Fig. 3).

Western blot analysis (Fig. $3 A$ ) including densitometric analysis (Fig. 3B) and immunocytochemistry for MBP (Fig. 3C) indicate that BDNF promoted myelination of wild-type mouse NGFdependent DRG neurons by rat Schwann cells. In contrast, BDNF had no effect on myelination of NGF-dependent neurons from p75NTR-/- mice (Fig. 3D-F). These data indicate that neuronal expression of p75NTR is necessary for the promyelinating influence of BDNF on these neurons.

To investigate whether Schwann cell p75NTR also plays a role in promoting myelination, we used a lentiviral-based shRNA ap- proach for the specific knockdown of p75NTR. Knockdown of p75NTR expression was observed in parallel Schwann cell cultures (Fig. 3G) and was not effected by BDNF treatment (Fig. $3 H$ ). BDNF enhanced myelination of NGF-dependent DRG neurons by noninfected (control), scrambled shRNA-infected and p75NTR-shRNA-infected Schwann cells, as assessed by immunocytochemistry (Fig. 3I) and Western blot analysis (Fig. 3J, K). In addition, in the untreated condition $(-)$, no difference in the basal level of myelination was observed between groups (supplemental Fig. 4, available at www.jneurosci.org as supplemental material). Collectively, these data suggest that Schwann cell expression of $\mathrm{p} 75 \mathrm{NTR}$ is not required for the promyelinating influence of BDNF.

\section{BDNF inhibits myelination of BDNF-dependent neurons via a neuronal mechanism}

We next used Campenot chambers to investigate the inhibitory effect BDNF exerts on BDNF-dependent neuron myelination (Fig. 4). Compared with control cultures (condition 1), addition of BDNF to the Axon+SC chamber (condition 2) reduced the number of myelin segments formed, whereas TrkB-Fc increased it (condition 3). Importantly, BDNF added to the DRG soma compartment reduced myelin segment formation (condition 4), indicating BDNF is acting neuronally to inhibit myelination. TrkB-Fc added to the DRG soma compartment did not alter myelination (condition 5). Thus, BDNF inhibits myelination of BDNF-dependent neurons by acting via neuronally expressed receptors.

\section{Activation of neuronal TrkB-FL inhibits myelination}

BDNF-dependent DRG neurons express four BDNF receptors: p75NTR, TrkB-FL, TrkB-T1, and TrkB-T2 (supplemental Fig. 2, available at www.jneurosci.org as supplemental material). As TrkB-FL mediates the majority of BDNF's functions, we next 

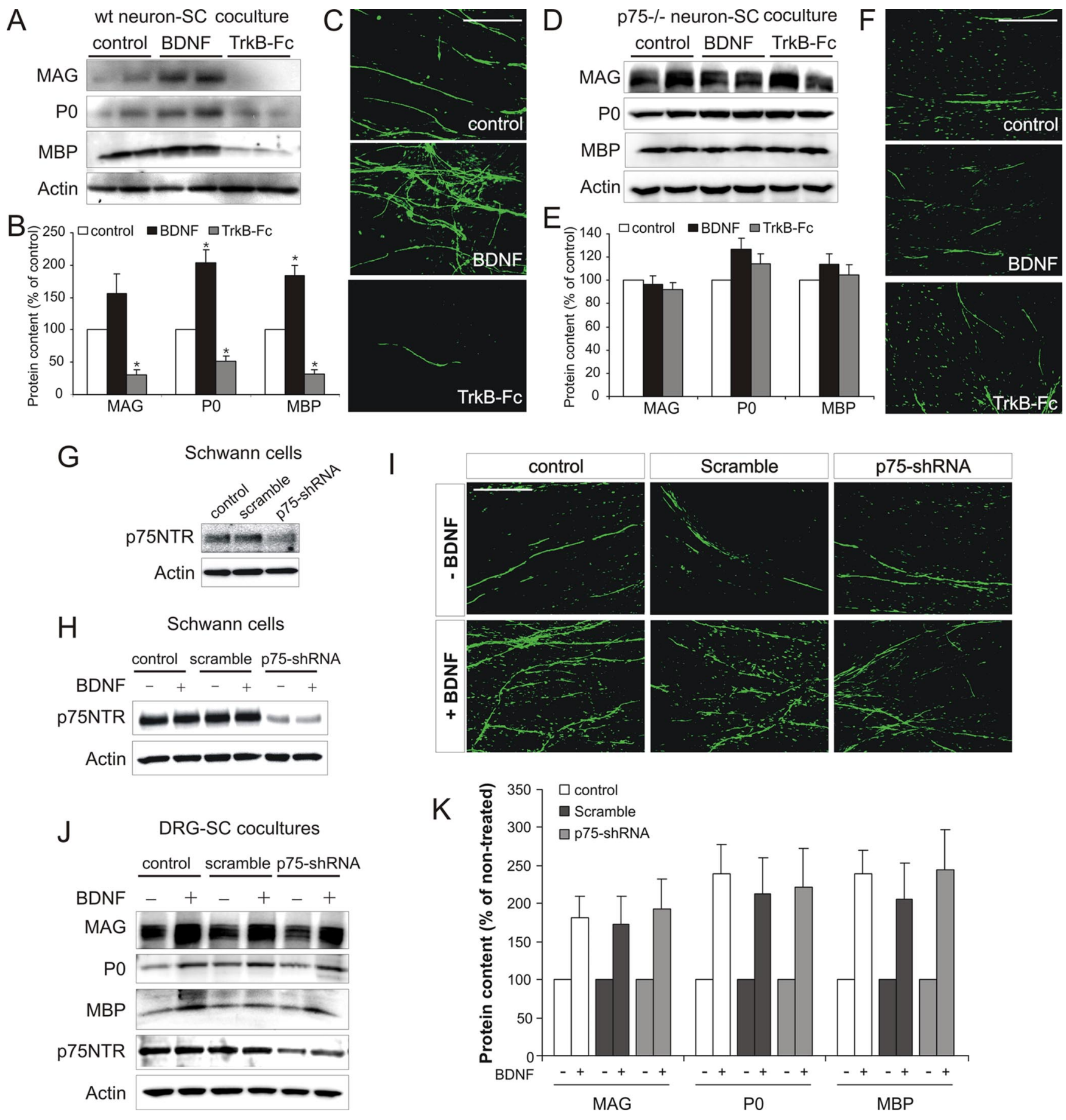

Figure 3. BDNF activates neuronal p75NTR receptors to promote myelination. $\boldsymbol{A}, \boldsymbol{B}$, Western blot $(\boldsymbol{A})$ including densitometric analysis $(\boldsymbol{B} ;$ mean $\pm \mathrm{SEM}, n=3)$ of cocultures containing NGF-dependent neurons from wild-type mice. BDNF increased myelin protein expression, whereas TrkB-Fcinhibited it $\left({ }^{*} p<0.01\right.$ vs control). C, MBP immunostaining of parallel cultures. Compared with controls, a qualitative increase in MBP + myelin segments occurred with BDNF but was reduced with TrkB-Fc. Scale bar, $100 \mu \mathrm{m} . \boldsymbol{D}$, $\boldsymbol{E}$, Western blot (D) including densitometric analysis $(\boldsymbol{E}$; mean \pm SEM,$n=3$ ) of cocultures containing NGF-dependent neurons from p75NTR $-/-$ mice. Neither BDNF nor TrkB-Fc altered myelin protein expression. $F$, MBP immunostaining of parallel p75NTR - / - cultures. No qualitative change in MBP + myelin segments was observed with either BDNF or TrkB-Fc. Scale bar, $100 \mu \mathrm{m}$. G-K, BDNF enhances myelination after p75NTR knockdown in Schwann cells. G, Western blot of noninfected (control), scrambled shRNA-infected, or p75NTR-shRNA-infected Schwann cell cultures. Knockdown of p75NTR expression was only observed in p75NTR-shRNA-infected cultures. $\boldsymbol{H}$, BDNF had no effect on p75NTR expression in either control, scramble-infected, or p75NTR-shRNA-infected cultures. $\boldsymbol{I}-\boldsymbol{K}, \mathbf{M B P}$ immunostaining $(\boldsymbol{I})$ and Western blot $(\boldsymbol{J})$ including densitometric analysis $(\boldsymbol{K} ;$ mean $\pm \mathrm{SEM}, n=3)$ of cocultures containing control, scramble-infected, or p75NTR-shRNA-infected Schwann cells. No qualitative change in MBP+ myelin segments or quantitative change of myelin protein expression occurred as a consequence of p75NTR knockdown. Scale bar, $100 \mu \mathrm{m}$.

examined the influence of BDNF on myelination in the presence of the Trk receptor inhibitor K252a (Knüsel and Hefti, 1992). In the cocultures, BDNF inhibited myelin protein expression, whereas K252a alone increased expression of P0 and MBP, as assessed by densitometric analysis of Western blots (Fig. 4C) and qualitative analysis of MBP + myelin segment formation (Fig. $4 D)$. Importantly, when K252a and BDNF were added concurrently $(\mathrm{K}+\mathrm{B}), \mathrm{K} 252 \mathrm{a}$ blocked the inhibitory effect of BDNF. As Schwann cells do not express TrkB-FL, these data implicate neuronal TrkB-FL activation mediating the inhibitory influence of 
BDNF on myelination of BDNFdependent DRG neurons. Interestingly, $\mathrm{K}+\mathrm{B}$ trended to increase myelination above the levels of K252a alone. This could suggest that inhibition of TrkB-FL signaling by K252a allows activation of p75NTR signaling by BDNF, resulting in a promyelinating influence. However, this is yet to be definitively established, and further analyses are required to investigate this point.

To further investigate the inhibitory effect of neuronal TrkB-FL receptor activation, we used K252a in Campenot chamber experiments (Fig. 4E,F). Compared with control cultures (condition 1), addition of BDNF to the Axon + SC compartment inhibited myelination (condition 2). $\mathrm{K} 252 \mathrm{a}$ added to the neuronal compartment enhanced myelin formation (condition 3). Importantly, when BDNF was added to the Axon + SC compartment and K252a concurrently added to the neuronal compartment (condition 4), the inhibitory effect of BDNF remained blocked. This shows that the selective inhibition of neuronal TrkB-FL receptor activation is sufficient to block the inhibitory BDNF signal, further implicating neuronal TrkB-FL receptors as mediating the inhibitory effect on myelination of BDNFdependent DRG neurons. Again, we observed a trend for the concurrent use of BDNF and K252a to increase myelination above K252a alone, further suggesting that when neuronal TrkB-FL signaling is inhibited, BDNF can exert a promyelinating influence via p75NTR.

\section{Discussion}

We have identified that BDNF exerts contrasting influences on the myelination of distinct DRG neuronal subpopulations. Using in vitro myelination studies, we have demonstrated that BDNF exerts a promyelinating influence on NGF-dependent DRG neurons by regulating the axonal signals that control myelination. Using neurons isolated from the p75NTR knock-out mouse, we have identified that BDNF acts in a p75NTR-dependent manner to regulate these axonal signals. We also identified a complementary role for BDNF as an inhibitor of myelination of BDNFdependent DRG neurons; this influence is also mediated by axonal signals but is ameliorated by pharmacological inhibition of tyrosine kinase signaling, suggesting a TrkB-mediated effect.

Neurotrophins have an established role in regulating peripheral myelination; however, the influence the different neurotrophins exert on the various subpopu-
A

TrkB+ DRG-SC cocultures in Campenot chambers
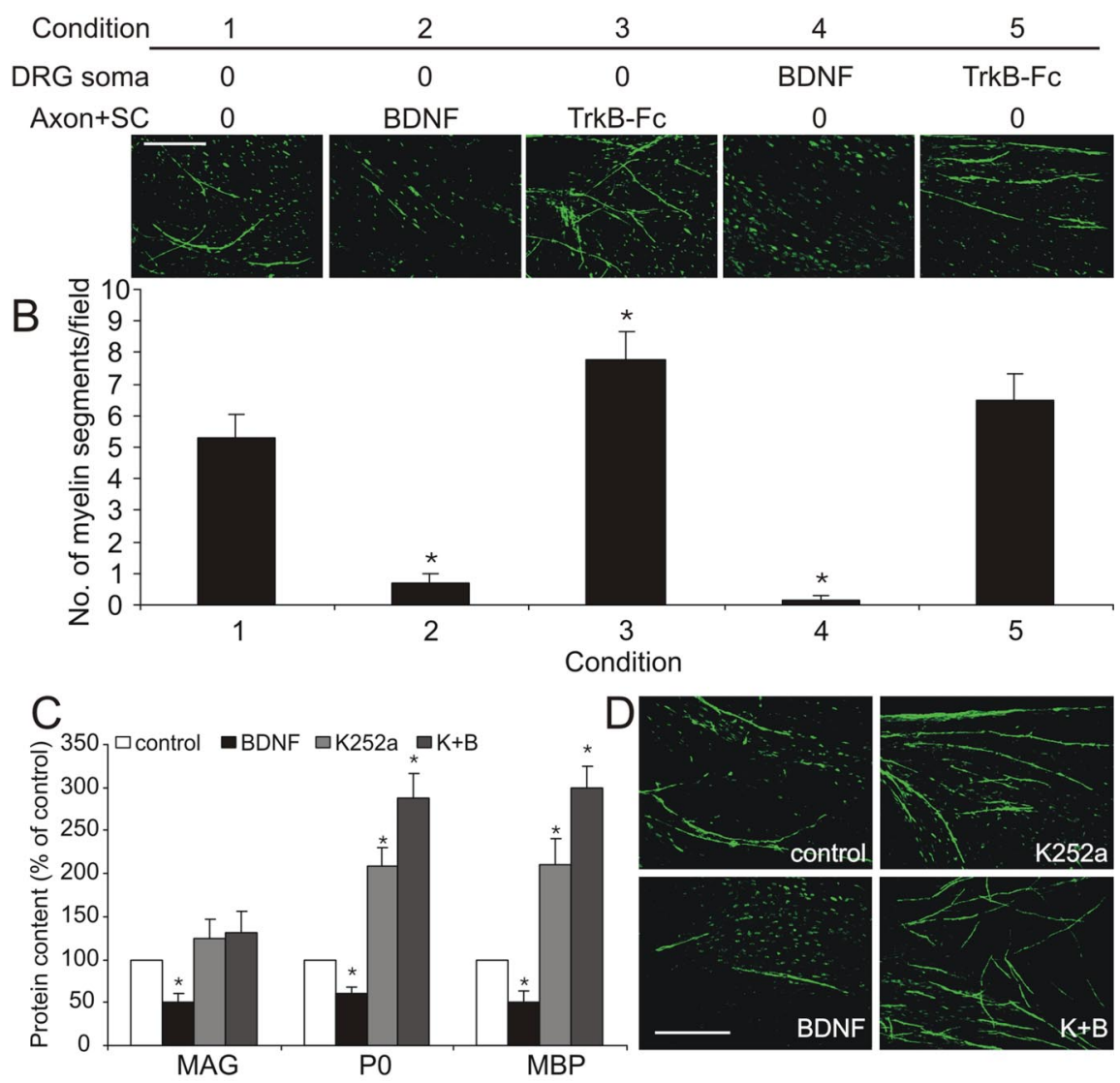

E TrkB+ DRG-SC cocultures in Campenot chambers



Figure 4. BDNF acts neuronally to inhibit myelination of BDNF-dependent neurons. $A, B$, Qualitative $(A)$ and quantitative $(B)$ analysis (4 fields per chamber, 3 chambers per condition) of MBP + myelin segments in Campenot chamber cocultures containing BDNFdependent neurons (mean \pm SEM, $n=3$ ). Compared with controls (condition 1), BDNF applied to the Axon +SC compartment (condition 2) inhibited, whereas TrkB-Fc (condition 3) increased myelination. When applied to the DRG soma compartment, BDNF inhibited myelination (condition 4), whereas TrkB-Fc had no effect (condition 5) ( ${ }^{*} p<0.05$ vs control). Scale bar, $100 \mu \mathrm{m}$. C, D, Densitometric analysis ( $\boldsymbol{C}$ of Western blot bands (mean $\pm \mathrm{SEM}, n=3$ ) and qualitative MBP + immunostaining (D) of BDNF-dependent cocultures. Exogenous BDNF inhibited myelin protein expression. K252a (200 nm) increased the expression of $\mathrm{PO}$ and MBP and the number of myelin segments. BDNF was unable to inhibit myelination in the presence K252a (B+K) ( ${ }^{*} p<0.05$ vs control). Scale bar, $100 \mu \mathrm{m} . \boldsymbol{E}, \boldsymbol{F}$, Qualitative $(\boldsymbol{E})$ and quantitative $(\boldsymbol{F})$ analysis of MBP + myelin segments after K252a (200 nM) treatment in Campenot chamber cocultures containing BDNF-dependent neurons (4 fields per chamber, 3 chambers per condition; mean $\pm \mathrm{SEM}, n=3)$. BDNF ( $100 \mathrm{ng} / \mathrm{ml}$ ) applied to the Axon + SC compartment (condition 2) inhibited, whereas K252a applied to the DRG soma compartment (condition 3) enhanced myelination. BDNF applied to the Axon + SC compartment was unable to inhibit the enhanced myelination with the concurrent addition of K252a to the DRG soma compartment (condition 4) ( ${ }^{*} p<0.05$ vs control). Scale bar, $100 \mu \mathrm{m}$. 
lations of DRG neurons remains uncertain. In vitro analysis of NGF-dependent DRG neurons demonstrated that increased expression of BDNF correlated with myelin production and identified that exogenous BDNF exerts a promyelinating influence (Chan et al., 2001). This is supported in vivo; BDNF injection into the sciatic nerve enhances peripheral myelin formation during development (Chan et al., 2001), and transgenic overexpression of BDNF increases both the rate and extent of peripheral myelination (Tolwani et al., 2004). Furthermore, BDNF is necessary for remyelination after peripheral nerve injury (Zhang et al., 2000). Although p75NTR has been shown to be critical for these effects (Cosgaya et al., 2002), identification of the cell type mediating this effect has remained elusive. The recent finding that BDNF induces polarity of Par-3 to the inner Schwann cell membrane adjacent to the axon during ensheathment suggested at least part of the promyelinating influence of BDNF is mediated via Schwann cells (Chan et al., 2006). However, by using p75NTR knock-out neurons in in vitro myelination assays, we have unambiguously identified that neuronal p75NTR receptors exert a necessary role in effecting the promyelinating influence of BDNF on the NGF-dependent subset of DRG neurons. Interestingly, after lentiviral mediated knockdown of p75NTR in Schwann cells, BDNF maintained its capacity to enhance myelination, suggesting Schwann cell p75NTR plays a limited role in myelination under these in vitro circumstances, further supporting the importance of neural signaling in this context. There also appears to be a fundamental difference in how NGF and BDNF promote Schwann cell myelination. NGF promotes the myelination of NGF-dependent neurons via activation of neuronally expressed TrkA receptors (Chan et al., 2004), whereas our data indicate that BDNF promotes myelination of these neurons via activation of neuronal p75NTR receptors.

Previous studies identifying a promyelinating role for BDNF were confined to analysis of NGF-dependent neurons (Chan et al., 2001; Cosgaya et al., 2002), but using BDNF-dependent DRG neurons, our data establish that BDNF can also exert an inhibitory role. At first pass, this might appear counterintuitive given that BDNF injection into the sciatic nerve enhances myelination during early postnatal development (Chan et al., 2001). However, TrkB is expressed in a small proportion ( $\sim 20 \%)$ of DRG neurons at this time (Wright and Snider, 1995); thus, in the presence of a promyelinating response by the majority population of TrkA+I p75NTR + DRG neurons, the effect that BDNF exerts on the TrkB + subpopulation would be difficult to identify. The response could also be developmental-stage specific. Clearly, it is important to establish whether BDNF exerts selective inhibitory effects on TrkB + DRG neurons in vivo. This is technically challenging but could be addressed using a transgenic approach to selectively prelabel TrkB-FL + DRG neurons and injecting BDNF into the developing sciatic nerve to establish an influence on myelination of these prelabeled neurons.

In combination, these data highlight a significant difference in the influence that Trk receptor activation exerts on peripheral myelination. NGF signaling through neuronal TrkA promotes Schwann cell myelination (Chan et al., 2004), whereas our data indicate that BDNF signaling through neuronal TrkB inhibits it. Given the structural and signaling similarities between TrkA and TrkB, precisely how this contrasting influence on myelination is mediated remains unclear. Nevertheless, TrkA and TrkB are expressed in phenotypically distinct subpopulations of DRG neurons and are rarely coexpressed in the same neuron (Wright and Snider, 1995). Thus, cell-context specificity in receptor tyrosine kinase signaling could account for these differential effects. Alter- natively, BDNF-induced signaling via TrkB-FL could be modified by truncated TrkB receptors.

Our data underscore the prominent role that neuronally derived signals exert in directing myelination. It is firmly established that axonal-derived neuregulin signaling exerts contextdependent effects at multiple stages of Schwann cell development (Michailov et al., 2004; Nave and Salzer, 2006). In particular, neuregulin-1 type III provides the key instructive signal that determines the ensheathment fate of axons (Taveggia et al., 2005). Interestingly, it has been demonstrated that both NGF and BDNF not only promote expression of neuregulin-1 in NGF-dependent DRG neurons in vitro but also induce its rapid cleavage and release as an essential component of its activation (Esper and Loeb, 2004). As Schwann cells express and secrete neurotrophins (Friedman et al., 1996), these cells could, therefore, directly influence the amount of neuregulin-1 stimulation they receive via selective targeting of neurotrophins to adjacent axons. Thus, although neuregulin-1 type III remains the ultimate arbiter of ensheathment fate, developmental changes in the expression, location, and activation of the neurotrophins and their receptors could induce Schwann cell-to-axon interactions critical for coordinated peripheral nerve myelination. The p75NTR and TrkA receptors are expressed in $\sim 80 \%$ of neurons in the early postnatal period (Molliver and Snider, 1997; Molliver et al., 1997), coincident with the commencement of myelination. Interestingly, TrkA expression is ultimately downregulated to $\sim 40 \%$ of DRG neurons by P14 (Molliver et al., 1995; Molliver and Snider, 1997), which are predominantly but not exclusively small and unmyelinated, whereas those that transiently express TrkA must compromise a significant component of the total myelinated pool of axons in the adult. Ultimately, the likelihood of an individual axon becoming myelinated is dependent on the orchestration of several independent factors that influence neuregulin-1 type III expression and processing, rather than Trk or p75NTR-mediated signaling in isolation. Contextual influences could also be germane to the inhibitory effect that BDNF exerts on the TrkB+ population, whereby the in vivo effect could be to delay rather than to block myelination and possibly to do so at critical stages of development when synaptogenesis and neuronal pruning are being effected. Resolution of these issues clearly requires detailed in vivo analysis, but this will be challenging given that neurotrophin and neurotrophin receptor-deficient mice all display severe deficits in DRG neuron survival, an important confounder (Conover and Yancopoulos, 1997).

Our findings contribute important insights into the influence BDNF exerts on peripheral myelination. First, neuronal p75NTR expression is critical for the promyelinating influence of BDNF. Second, BDNF-induced activation of neuronal TrkB receptors inhibits Schwann cell myelination. Neurotrophin-mediated influences on the capacity of individual axons to become myelinated are, therefore, determined by the unique cell-specific expression of neurotrophin receptors. These findings also highlight the importance of a thorough examination of the influence of putative remyelinatitive therapies on all DRG neuronal subpopulations rather than assuming a unified response.

\section{References}

Bentley CA, Lee KF (2000) p75 is important for axon growth and schwann cell migration during development. J Neurosci 20:7706-7715.

Campenot RB (1992) Cell-cell interactions: a practical approach. Oxford: IRL.

Chan JR, Cosgaya JM, Wu YJ, Shooter EM (2001) Neurotrophins are key mediators of the myelination program in the peripheral nervous system. Proc Natl Acad Sci U S A 98:14661-14668. 
Chan JR, Watkins TA, Cosgaya JM, Zhang C, Chen L, Reichardt LF, Shooter EM, Barres BA (2004) NGF controls axonal receptivity to myelination by Schwann cells or oligodendrocytes. Neuron 43:183-191.

Chan JR, Jolicoeur C, Yamauchi J, Elliott J, Fawcett JP, Ng BK, Cayouette M (2006) The polarity protein Par-3 directly interacts with p75NTR to regulate myelination. Science 314:832-836.

Conover JC, Yancopoulos GD (1997) Neurotrophin regulation of the developing nervous system: analyses of knockout mice. Rev Neurosci 8:13-27.

Cosgaya JM, Chan JR, Shooter EM (2002) The neurotrophin receptor p75NTR as a positive modulator of myelination. Science 298:1245-1248.

Esper RM, Loeb JA (2004) Rapid axoglial signaling mediated by neuregulin and neurotrophic factors. J Neurosci 24:6218-6227.

Friedman HC, Jelsma TN, Bray GM, Aguayo AJ (1996) A distinct pattern of trophic factor expression in myelin-deficient nerves of Trembler mice: implications for trophic support by Schwann cells. J Neurosci 16:5344-5350.

Greferath U, Bennie A, Kourakis A, Bartlett PF, Murphy M, Barrett GL (2000) Enlarged cholinergic forebrain neurons and improved spatial learning in p75 knockout mice. Eur J Neurosci 12:885-893.

Höke A, Ho T, Crawford TO, LeBel C, Hilt D, Griffin JW (2003) Glial cell line-derived neurotrophic factor alters axon schwann cell units and promotes myelination in unmyelinated nerve fibers. J Neurosci 23:561-567.

Huang EJ, Reichardt LF (2001) Neurotrophins: roles in neuronal development and function. Annu Rev Neurosci 24:677-736.

Ishibashi T, Dakin KA, Stevens B, Lee PR, Kozlov SV, Stewart CL, Fields RD (2006) Astrocytes promote myelination in response to electrical impulses. Neuron 49:823-832.

Knüsel B, Hefti F (1992) K-252 compounds: modulators of neurotrophin signal transduction. J Neurochem 59:1987-1996.

Lee KF, Li E, Huber LJ, Landis SC, Sharpe AH, Chao MV, Jaenisch R (1992) Targeted mutation of the gene encoding the low affinity NGF receptor p75 leads to deficits in the peripheral sensory nervous system. Cell 69:737-749.

Michailov GV, Sereda MW, Brinkmann BG, Fischer TM, Haug B, Birchmeier C, Role L, Lai C, Schwab MH, Nave KA (2004) Axonal neuregulin-1 regulates myelin sheath thickness. Science 304:700-703.
Molliver DC, Snider WD (1997) Nerve growth factor receptor TrkA is down-regulated during postnatal development by a subset of dorsal root ganglion neurons. J Comp Neurol 381:428-438.

Molliver DC, Radeke MJ, Feinstein SC, Snider WD (1995) Presence or absence of TrkA protein distinguishes subsets of small sensory neurons with unique cytochemical characteristics and dorsal horn projections. J Comp Neurol 361:404-416.

Molliver DC, Wright DE, Leitner ML, Parsadanian AS, Doster K, Wen D, Yan Q, Snider WD (1997) IB4-binding DRG neurons switch from NGF to GDNF dependence in early postnatal life. Neuron 19:849-861.

Nave KA, Salzer JL (2006) Axonal regulation of myelination by neuregulin 1. Curr Opin Neurobiol 16:492-500.

Ng BK, Chen L, Mandemakers W, Cosgaya JM, Chan JR (2007) Anterograde transport and secretion of brain-derived neurotrophic factor along sensory axons promote Schwann cell myelination. J Neurosci 27:7597-7603.

Song XY, Zhou FH, Zhong JH, Wu LL, Zhou XF (2006) Knockout of p75(NTR) impairs re-myelination of injured sciatic nerve in mice. J Neurochem 96:833-842.

Taveggia C, Zanazzi G, Petrylak A, Yano H, Rosenbluth J, Einheber S, Xu X, Esper RM, Loeb JA, Shrager P, Chao MV, Falls DL, Role L, Salzer JL (2005) Neuregulin-1 type III determines the ensheathment fate of axons. Neuron 47:681-694.

Tolwani RJ, Cosgaya JM, Varma S, Jacob R, Kuo LE, Shooter EM (2004) BDNF overexpression produces a long-term increase in myelin formation in the peripheral nervous system. J Neurosci Res 77:662-669.

Woolley AG, Tait KJ, Hurren BJ, Fisher L, Sheard PW, Duxson MJ (2008) Developmental loss of NT-3 in vivo results in reduced levels of myelinspecific proteins, a reduced extent of myelination and increased apoptosis of Schwann cells. Glia 56:306-317.

Wright DE, Snider WD (1995) Neurotrophin receptor mRNA expression defines distinct populations of neurons in rat dorsal root ganglia. J Comp Neurol 351:329-338.

Zhang JY, Luo XG, Xian CJ, Liu ZH, Zhou XF (2000) Endogenous BDNF is required for myelination and regeneration of injured sciatic nerve in rodents. Eur J Neurosci 12:4171-4180. 